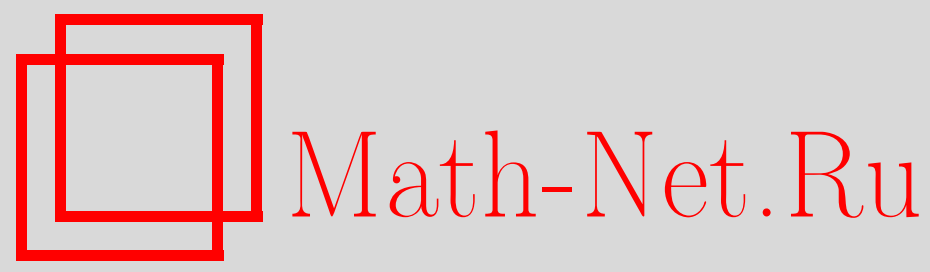

В. А. Орлов, О реализации $k$-значных функций схемами из функциональных элементов, Матем. заметки, 1998, том 64, выпуск 3, 431-436

DOI: https://doi.org/10.4213/mzm1414

Использование Общероссийского математического портала Math-Net.Ru подразумевает, что вы прочитали и согласны с пользовательским соглашением http://www . mathnet.ru/rus/agreement

Параметры загрузки:

IP : 54.205 .225 .156

26 апреля 2023 г., 13:43:25

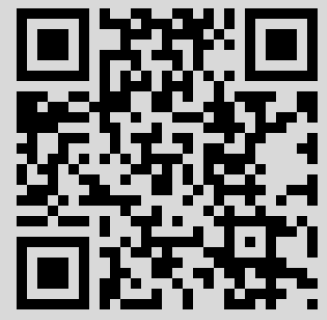


УДК 519.6

\title{
О РЕАЛИЗАЦИИ $k$-ЗНАЧНЫХ ФУНКЦИЙ СХЕМАМИ ИЗ ФУНКЦИОНАЛЬНЫХ ЭЛЕМЕНТОВ
}

\author{
В.А. Орлов
}

Рассматриваются вопросы реализации функций из $P_{k}$ схемами из функциональных элементов в произвольных базисах.

Библиограбфия: 4 названия.

Пусть $\Phi$ - произвольная полная в $P_{k}(k \geqslant 2)$ система функций, каждая из которых существенно зависит от конечного числа всех своих переменных. Системе $\Phi$ сопоставим базис Б, состоящий из реализующих ее функции элементов, каждому из которых приписано положительное число (вес элемента). Для имеющего не менее двух входов элемента его приведенным весом [1] назьвается отношение веса к уменьшенному на единицу числу входов. Приведенным весом $\rho_{\text {Б }}$ базиса Б назьвается минимум приведенных весов его элементов. Всюду в дальнейшем будем рассматривать схемы в базисе Б и под $\rho$ понимать $\rho_{\mathrm{Б}}$.

Под слохнностью $L(S)$ схемы $S$ будем понимать сумму весов ее элементов. Через $L(G)$ обозначается минимальная сложность схемы, реализующей систему функций $G$, а через $L(k, n, r)$ - максимум $L(F)$ по всем системам $F$ из $r$ функций из $P_{k}$ от $n$ переменных.

О.Б. Лупанов [1], [2] решил задачу нахождения асимптотики функции Шеннона $L(2, n, r)$.

Предлагается метод реализации функций из $P_{k}$ для любого $k \geqslant 2$. С использованием этого метода показано, что для почти любого базиса в $P_{k}$ асимптотическое поведение функции Шеннона определяется его минимальньг приведенным весом.

Отметим, что с использованием этого метода в [3], [4] для любого $k>3$ показано существование базисов, в которых асимптотическое поведение функции Шеннона зависит от параметров многих элементов.

Пусть

$$
\rho(n)=\min _{f} \frac{L(f)}{n-1}
$$

по всем функциям $f$, существенно зависящим от $n$ переменных, и пусть $\rho^{*}=\lim _{n \rightarrow \infty} \rho(n)$. Докажем существование этого предела.

Имеющая $n$ входов и $m$ выходов схема назьвается $(n, m)$-блоком.

Пусть $h_{p}$ - существенно зависящая от $p$ переменных функция из $P_{k}$ такая, что $L\left(h_{p}\right)=$ $\rho(p)(p-1)$, и пусть $H_{p}$ - реализуюший эту функцию $(p, 1)$-блок.

Пусть $S$ - схема, состоящая из $m$ блоков $H_{n}$ и $(m+r, 1)$-блока $M$, реализующего функцию $\max \left(z_{1}, \ldots, z_{m+r}\right)$. Входами (выходом) этой схемы являются входы схем $H_{n}$ (выход блока $M$ ). Выходы блоков $H_{n}$ соединены со входами блока $M$. Нетрудно проверить, что схема $S$ реализует функцию, существенно зависящую от всех своих $m n+r$ переменных. Таким образом, при любых $n, m$ и $r(r<n)$ имеет место соотношение 
$\rho(n m+r)<\rho(n)+c / n+c / m$, доказьвающее единственность предела (с использованием нижнего предела).

Отметим теперь, что $\rho^{*} \geqslant \rho$ и $L\left(H_{p}\right) \sim \rho^{*} p$.

Верна следующая

TEOpEMA 1. $L(k, n, 1) \leqslant \rho^{*} k^{n} / n$.

Доказательство этой теоремы будет проведено ниже с использованием прини ипа глобального кодирования, заключающегося в представлении функции из $P_{k}$ системой булевых функций.

Вначале опишем несколько вспомогательных блоков и докажем теорему о реализации булевых функций.

Пусть $d_{i, 1}, \ldots, d_{i, i-1}, d_{i, i+1}, \ldots, d_{i, p}(1 \leqslant i \leqslant p)$ - набор значений переменных, обеспечивающий существенность $i$-й переменной функции $h_{p}$, и пусть $d_{i, i}$ и $e_{i}-$ значения $i$-й переменной, на которых функция $h_{p}$ при этом принимает различные значения $a_{i}$ и $b_{i}$. Пусть $b_{p, i}-$ булев набор длины $p$, имеющий ровно одну $i$-ю единичную компоненту. Пусть $H S C_{p}-(p, p+2)$-блок, который на наборе $b_{p, i}(1 \leqslant i \leqslant p)$ выдает набор $d_{i, 1}, \ldots, d_{i, p}, e_{i}, b_{i}$. Нетрудно получить оценку $L\left(H S C_{p}\right)<c p^{2}$. Здесь и далее $c-$ некоторая, зависящая от базиса, константа.

Пусть $B C(B D)-(2,1)$-блок, реализующий функцию, равную 1 на наборе 1, 1 (равную 0 на наборе 0,0$)$ и равную $0(1)$ на остальных наборах. Эти функции будем назьвать конғюнкиией и дизбюнкиией и обозначать через \& и $\vee$. Через $B D_{r}(r \geqslant 3)$ будем обозначать $(r, 1)$-блок, реализуюший функцию $z_{1} \vee \cdots \vee z_{r}$.

Пусть $S L-(3,1)$-блок, реализующий функцию, равную $z_{1}$ при $z_{3}=0$ и равную $z_{2}$ в остальных случаях.

Пусть $E Q-(2,1)$-блок, реализующий функцию, равную 1 при $z_{1}=z_{2}$ и равную 0 в остальных случаях.

Через $\mathrm{lb}(x)$ будем обозначать $\log _{2} x$.

В соответствии с принципом глобального кодирования рассмотрим реализацию булевых функций схемами в базисах из $P_{k}$.

ТЕОРема 2. Для произвольной булевой функиии $f$ от $n$ переменных выполнено $L(f) \leqslant \rho^{*} 2^{n} / n$.

ДокАЗАТЕЛЬСтво. Используем первое представление булевых функций $[1$, с. 68$]$. А именно, вводим параметры $r$ и $s$ и задаем $f$ булевой $\left(2^{r}, 2^{n-r}\right)$-матрицей $M^{f}$, которую разбиваем на полосы $M_{i}^{f}$, каждая из которых, кроме, быть может, последней, содержит $s$ строк. Число полос $p=] 2^{r} / s[$.

В соответствии с этим переменные функции $f$ разобьем на две грушы $x_{1}, \ldots, x_{r}$ и $y_{1}, \ldots, y_{n-r}$. Через $X(Y)$ будем обозначать набор $x_{1}, \ldots, x_{r}$ (набор $\left.y_{1}, \ldots, y_{n-r}\right)$. Через $X_{j}\left(Y_{j}\right)$ будем обозначать набор $X(Y)$, являющийся двоичной записью числа $j$. Таким образом, значение $f\left(X_{i}, Y_{j}\right)$ является элементом $M_{i, j}^{f}$.

Пусть $U_{i, t}(X, Y)$ - функция, все столбцы $i$-й полосы матрицы которой являются двоичной записью числа $t$, а все остальные строки нулевые.

Пусть $C X_{j}(X)$ (соответственно $\left.C Y_{j}(Y)\right)$ - функция, равная 1 на наборе $X_{j}$ (на набоpe $\left.Y_{j}\right)$ и равная 0 на остальных наборах.

Пусть $t_{i, j}^{f}$ - число, двоичной записью которого является $j$-й столбец полосы $M_{i}^{f}$. Через $f_{i, j}(X, Y)$ будем обозначать $U_{i, t}(X, Y)$ при $t=t_{i, j}^{f}$, а через $f_{j}(X, Y)-d p\left(f_{1, j}(X, Y)\right.$, $\left.\ldots, f_{p, j}(X, Y)\right) \& C Y_{j}(Y)$, где $d p\left(z_{1}, \ldots, z_{p}\right)$ - функция, равная 0 на наборе $(0, \ldots, 0)$, равная 1 на всех булевых наборах, имеющих ровно одну единицу, и принимающая любые 
значения на остальных наборах.

Теперь функцию $f$ будем реализовывать в соответствии с разложением $f(X, Y)=$ $f_{0}(X, Y) \vee \cdots \vee f_{v-1}(X, Y)$. Здесь и далее $v=2^{n-r}$.

Реализующая функцию $f$ схема $S$ состоит из $\left(r, 2^{r}\right)$-блока $C X,(n-r, v)$-блока $C Y$, $\left(2^{r}, u\right)$-блока $U B,(3 u, u+1)$-блока $C P U,(u+1, v)$-блока $B S,(2 v, v)$-блока $C O N$ и блока $B D_{v}$. Здесь и далее $u=p 2^{s}$.

Блок $C X(C Y)$ реализует все функции $C X_{i}(X)\left(C Y_{j}(Y)\right)$. Входы этих блоков являются входами схемы $S$.

Блок $U B$, используя функции $C X_{i}(X)$, реализует все функции $U_{i, t}(X, Y)$.

Блок $C P U$ состоит из $u$ блоков $S L$ и блока $H S C_{p}$. Входами блока $C P U$ являются третьи входы его блоков $S L$. Они соединены с выходами блока $U B$. Входы блока $H S C_{p}$ соединены с выходами блока $U B$, соответствующими характеристическим функциям полос матрицы $M^{f}$ (функциям $U_{i, t}$ при $t=2^{s}-1$ ). Первьй (второй) вход соответствующего функции $U_{i, t}$ блока $S L$ соединен с $i$-м $\left((p+1)\right.$-м) выходом блока $H S C_{p} . \mathrm{B}$ блоке $C P U(u+1)$-м выходом является $(p+2)$-й выход блока $H S C_{p}$.

Блок $B S$ состоит из $v$ блоков $H_{p}$, входы которых являются его входами, и $v$ блоков $E Q$. Выход каждого блока $H_{p}$ соединен с первьм входом соответствующего ему блока $E Q$. Вторые входы блоков $E Q$ соединены с $(u+1)$-м входом блока $B S$, который соединен с $(u+1)$-м выходом блока $C P U$. В блоке $B S i$-й вход $j$-го блока $H_{p}$ соединен с выходом блока $C P U$, соответствующим функции $U_{i, t}$ при $t=t_{i, j}^{f}$. Выходами блока являются выходы блоков $E Q$.

Блок $C O N$ состоит из $v$ блоков $B C$. Первьй (второй) вход $j$-го блока $B C$ соединен с $j$-м выходом блока $B S$ (блока $C Y$ ).

Входы блока $B D_{v}$ соединены с выходами блока $C O N$, его выход является выходом схемы $S$.

Приведем известные (или легко получаемые) оценки сложностей блоков схемы $S$ :

$$
\begin{gathered}
L(K X)<c 2^{r}, \quad L(U B)(L(C P U))<c u \\
L(C Y)\left(L(C O N), L\left(B D_{v}\right)\right)<c v, \quad L(B S) \sim \rho^{*} p v .
\end{gathered}
$$

Отметим, что все блоки имеют линейную относительно суммы чисел их входов и выходов сложность. Параметры $r$ и $s$ выбираем так, чтобы основную сложность схемы $S$ составил блок $B S$. При $r=[2 \mathrm{lb}(n)]$ и $s=[n-3 \mathrm{lb}(n)]$ получаем утверждение теоремы 2 .

Через $q$ будем обозначать $\mathrm{lb}(k)$.

ДоКАЗАТЕЛЬСТВо ТЕОРЕмЫ 1 . Пусть сначала $q$ - целое число. Пусть $g_{n}$ - произвольная функция из $P_{k}$ от $n$ переменных. Опишем реализующую эту функцию схему $S$ и оценим ее сложность. Пусть $m=q n$. Схема $S$ состоит из $(n, m)$-блока $A,(m, q)$-блока $B$ и $(q, 1)$-блока $C$. Входами блока $A$ являются входы схемы $S$. Входы блока $B$ (блока $C$ ) соединены с выходами блока $A$ (блока $B$ ). Выходом схемы $S$ является выход блока $C$. Блок $A$ реализует двоичную запись числа, $k$-ичной записью которого является набор значений входов схемы $S$. По функции $g_{n}$ и блоку $A$ однозначно определяется система из $q$ булевых функций от $m$ переменных такая, что набор значений ее функций является двоичной записью значения функции $g_{n}$. Блок $B$ реализует эту систему булевых функций. Блок $C$ осуществляет восстановление значения функции $g_{n}$ по его двоичной записи.

Из оценок $L(A)<c q n, L(C)<c k$ и теоремы 2 следует утверждение теоремы 1 в случае, когда $k$ является степенью двойки. 
Если $q$ не является целым, то функцию $g_{n}$ разлагаем по $r=[\mathrm{lb}(n) / q]$ переменным и реализуем $k^{r}$ функций из $P_{k}$ от $n-r$ переменных с помощью системы из $q k^{r}$ [ булевых функций от $] q(n-r)$ [ переменных, определенных на первых $k^{n-r}$ наборах.

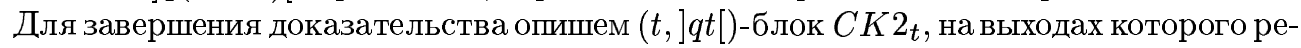
ализуется двоичная запись числа, $k$-ичной записью которого является его входной набор. Блок состоит из $t(1] q t[)$, -блоков $C R_{j}$ и $(t] q t[] q t[)$, -блока $S M$. Вход блока $C R_{j}$ является $j$-м входом блока $C K 2_{t}$, на его выходах реализуется двоичная запись числа $z_{j} k^{j-1}$, где $z_{j}$ - значение $j$-го входа блока $C K 2_{t}$. Блок $S M$ осуществляет последовательное суммирование этих чисел. Выходы блока $S M$ являются выходами блока $C K 2_{t}$. Нетрудно получить оценку $L\left(C K 2_{t}\right)<c t^{2}$. Аналогичньм образом строится (]$q t[, t)$-блок $C 2 K_{t}$, на выходах которого реализуется $k$-ичная запись числа, двоичной записью которого является его булев входной набор.

Отметим, что при обычных ограничениях имеет место соотношение

$$
L(k, n, r) \stackrel{\sim}{\leqslant} \rho^{*} \frac{r k^{n}}{n+\log _{k} r} .
$$

Базис Б назовем оптимальным, если $L(k, n, 1) \sim \rho k^{n} / n$.

Покажем оптимальность почти всех базисов.

Существенно зависящая не менее чем от двух переменных функция называется существенной. Существенную функцию, существенно зависящую от всех своих переменных, назовем $S$-функиией. Систему существенных функций из $P_{k}$ назовем сильно существенной, если для любого числа $m$ из этих функций можно построить являющуюся $S$-функцией бесповторную суперпозицию от $n>m$ переменных и если любая ее подсистема этим свойством не обладает. Образующую сильно существенную систему функцию назовем сильно существенной.

Систему функций всех, имеющих минимальньй приведенньй вес, элементов базиса назовем его ядром. Отметим, что ядро любого базиса не пусто. Базис назовем сильно существенным, если его ядро содержит сильно существенную систему. Отметим, что в случае сильно сушественных базисов $\rho^{*}=\rho$.

ТЕОрема 3. Сильно существенный базис оптимален.

Доказательство следует из теоремы 1 и из обычных мощностных соображений.

Заметим, что в случае сильно существенных базисов получаем порядок остаточного члена функции Шеннона $L(k, n, r)$.

Оценим число сильно существенных функций.

Всюду в дальнейшем, если не оговорено другое, под функцией будем понимать функцию из $P_{k}(k \geqslant 3)$ от $n$ переменных. Через $R_{f}$ будем обозначать множество значений функции $f$.

Через $f_{i, s}$ будем обозначать подфункцию, получающуюся из функции $f$ при значении $i$-й переменной, равном $s$.

$\Phi$ ункцию $f$ назовем $V_{i}$ такие, что $f_{i, s}=f_{i, t}$.

Функцию $f$ назовем $Z_{i, j}$ функиией $(j \neq i)$, если для любого $s$ из $R_{f}$ подфункция $f_{i, s}$ не зависит существенно от $j$-й переменной. $Ф$ ункцию, являющуюся $Z_{i, j}$-функцией при некотором $j$, назовем $Z_{i}$ функиией.

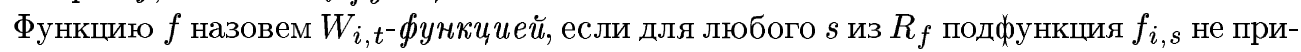
нимает значения $t$ из $R_{f}$. Функцию, являющуюся $W_{i, t}$-функцией при некотором $t$, назовем $W_{i}$-функиией. 


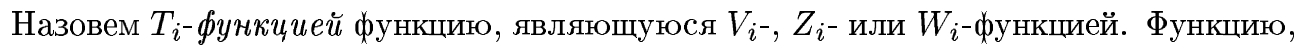
не являющуюся $T_{i}$-функцией, назовем $N T_{i}$ - функиией. Отметим, что $N T_{i}$-функция не является ни $V_{i}$-функцией, ни $Z_{i}$-функцией и ни $W_{i}$-функцией.

ТЕОРемА 4. S-функиия, являющаяся $N T_{i}$-функиией при некотором $i$, сильно существенна.

ДокАЗАТЕЛЬСТво. Не ограничивая общности, полагаем $i=n$. Пусть $f-$ произвольная удовлетворяющая условиям теоремы функция.

Из произвольного числа $t$ реализующих функцию $f$ элементов построим цепочку, в которой выход каждого элемента соединен с $n$-м входом последующего.

Покажем существенность $j$-го $(1 \leqslant j<n)$ входа $m$-го $(1 \leqslant m \leqslant t)$ элемента этой цепочки.

Так как $f$ не является $Z_{n}$-функцией, то существует $s$ из $R_{f}$ такое, что $f_{n, s}$ существенно зависит от $j$-й переменной. Функцию $f_{n, s}$ обозначим через $g$. Из существенности $j$-й переменной функции $g$ следует существование $a$ и $b$ таких, что $g_{j, a} \neq g_{j, b}$. Функцию $g_{j, a}\left(g_{j, b}\right)$ обозначим через $h_{a}\left(h_{b}\right)$. Таким образом, существует набор $Y_{m}$ значений, отличных от $n$-го и $j$-го входов $m$-го элемента, такой, что значения $c$ и $d$ функций $h_{a}$ и $h_{b}$ на этом наборе различны.

Так как $f$ не является $V_{n}$-функцией, то существует набор $Y_{m+1}$ значений, отличных от $n$-го входа $(m+1)$-го элемента, такой, что значения функций $f_{n, c}$ и $f_{n, d}$ на этом наборе различны. Аналогичным образом показьвается существование таких наборов для последующих элементов цепочки.

Так как $f$ не является $W_{n}$-функцией, то существует набор $Y_{m-1}$ значений входов, отличных от $n$-го, и значение $s_{m-1} n$-го входа $(m-1)$-го элемента такие, что значение выхода $(m-1)$-го элемента равно $s$. Аналогичным образом показьвается существование таких наборов и значений $n$-х входов для предыдущих элементов цепочки.

Доказательство существенности $n$-го входа первого элемента цепочки является более простьм случаем рассмотренного вьше.

Теорема 4 доказана.

Через $M_{r}$ будем обозначать множество функций, принимающих $r$ значений, через $S_{r}$ - множество $S$-функций из $M_{r}$, а через $N C_{r}$ - множество функций из $S_{r}$, не являющихся сильно существенными. Через $s_{r}\left(c_{r}\right)$ обозначим число функций из $S_{r}$ (из $\left.N C_{r}\right)$. Пусть $d_{r}=c_{r} / s_{r}$ и $k_{r}=r^{k^{n}} / s_{r}$. Из соотношения $s_{r} \geqslant r^{k^{n}}-n r^{k^{n-1}}-r(r-1)^{k^{n}}$ следует, что $k_{r}$ быстро стремится к 1 .

Оценим число функций из $N C_{r}$ и их долю $d_{r}$.

Функцию, являющуюся $T_{i}$-функцией для любого $i$, назовем $T$-функиией.

Из теоремы 4 следует

\section{Теорема 5. Число функций из $N C_{r}$ не превосходит числа T-функций.}

Не ограничивая общности, при проведении доказательства будем полагать, что функции из $M_{r}$ принимают первые $r$ значений.

Функцию, являющуюся $V_{i}$-функцией для любого $i$, назовем $V$-функиией.

Лемма 1. Число $V$-функиий из $M_{r}$ не превосходит $\left(C_{r}^{2}\right)^{n} r^{(k-1)^{n}}$.

ДокАЗАТЕЛЬСТВо. Пусть $s_{i}$ и $t_{i}(1 \leqslant i \leqslant n)$ - значения $i$-го аргумента из определения $V$-функции, и пусть $s_{i}<t_{i}$.

В таблище для функции $f$ удалим наборы, $i$-я компонента которых равна $t_{i}(1 \leqslant i \leqslant n)$. По содержащей $(k-1)^{n}$ наборов оставшейся части и парам $\left(s_{i}, t_{i}\right) \quad V$-функция восстанавливается полностью. Лемма доказана. 
ЛЕмма 2. Для любого $і$ число $Z_{i}$-функций из $M_{r}$ не превосходит

$$
(n-1) r^{k^{n}-r(k-1) k^{n-2}} \text {. }
$$

ДокАЗАТЕЛЬСтво. $Z_{i, j}$-функцию будем рассматривать как набор $k^{n-2}$ функций от двух переменных $(i$-й и $j$-й). Каждую такую функцию будем задавать $(k \times k)$-матрицей, в которой строки (столбцы) соответствуют значениям $i$-й $(j$-й) переменной. Во всех этих матрицах каждая из первых $r$ строк состоит из одинаковых элементов, т.е. эти матрицы задаются $k(k-r)+r$ элементами. Лемма доказана.

Через $m_{r}$ будем обозначать $(r /(r-1))^{r}$.

Легко проверить, что верна

Лемма 3. Для любого $i(1 \leqslant i \leqslant n)$ число $W_{i}$-функций из $M_{r}$ не превосходит $r^{k^{n}+1} m_{r}^{-k^{n-1}}$.

\section{Из лемм 1-3 следует}

Лемма 4. Число Т-функиий из $M_{r}$ не превосходит

$$
\left(C_{r}^{2}\right)^{n} r^{(k-1)^{n}}+n(n-1) r^{k^{n}-r(k-1) k^{n-2}}+n r^{k^{n}+1} m_{r}^{-k^{n-1}} .
$$

Отсюда и из теоремы 5 следует

Теорема 6. Справедлива оченка

$$
d_{r}<k_{r}\left(\left(C_{r}^{2}\right)^{n} r^{(k-1)^{n}-k^{n}}+n(n-1) r^{-r(k-1) k^{n-2}}+n r m_{r}^{-k^{n-1}}\right) .
$$

Таким образом, для любого $r$ доля существенно зависящих от всех $n$ своих переменных и принимаюших $r$ значений функций из $P_{k}$, не являющихся сильно существенными, дважды экспоненциально убьвает с ростом $n$ и более чем экспоненциально убывает с ростом $k$.

Отметим, что при $r=k$ (при $r=2$ ) в теореме 6 достаточно взять только первое слагаемое (два первых слагаемых). Кроме того, оценку можно улучшить, учитьвая

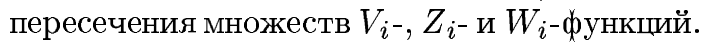

Базис с равным 1 приведенным весом назовем нормированным. Не ограничивая общности, будем рассматривать нормированные базисы. Для оценки доли сильно существенных базисов множество базисов разобьем на подмножества, элементы которых имеют одинаковые мошности их систем функций и ядер, и будем полагать эквивалентньми базисы, имеющие одинаковые системы функций и ядра.

Максимальное число входов элементов базиса назовем его рангом. Из теорем 3 и 6 следует, что при растущем ранге или значности почти все базисы в $P_{k}$ оптимальны.

\section{СПИСОК ЦИТИРОВАННОЙ ЛИТЕРАТУРЫ}

[1] Лупанов О.Б. О синтезе некоторых классов управляющих систем // Проблемы кибернетики. Вып. 10. М.: Физматгиз, 1963. С. 63-97.

[2] Лупанов О.Б. Об одном подходе к синтезу управляющих систем - принципе локального кодирования // Проблемы кибернетики. Вып. 14. М.: Физматгиз, 1965. С. 31-110.

[3] Орлов В.А. О реализации функций из $P_{k}$ схемами в произвольном базисе // ХІ Международная конференция по проблемам теоретической кибернетики. Тезисы докл. Ульяновск, 1996. C. $154-155$.

[4] Орлов В.А. Реализация функций из $P_{k}$ схемами в произвольном базисе из функциональных элементов // Докл. РАН. 1998. Т. 359. № 3. С. 308-309. 